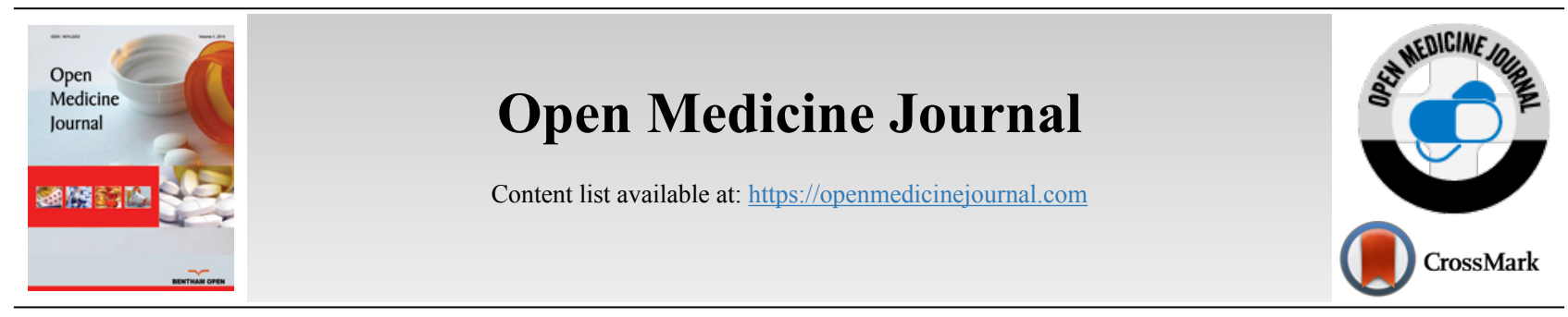

RESEARCH ARTICLE

\title{
T1 and T2 Mappings in the Early Diagnosis of Achilles Tendinosis
}

\author{
Zeineb Tbini ${ }^{1, *}$, Mokhtar Mars ${ }^{1}$ and Mouna Chelli ${ }^{2}$ \\ ${ }^{1}$ El Manar University, Higher Institute of Medical Technologies of Tunis, Laboratory of Research in Biophysics and Medical Technologies, 09, Rue \\ Doctor Zouheir Safi-1006 Tunis, Tunisia \\ ${ }^{2}$ Radiology Department, Mohamed Kassab Institute of Orthopedics, 2010 Ksar Saï, Manouba, Tunisia.
}

\begin{abstract}
:
Objective:

The purpose of this study was to compare T1 and T2 relaxation times of normal and pathologic Achilles Tendon (AT) in order to evaluate the ability of these methods to detect early Achilles tendon tendinosis.

\section{Materials and Methods:}

Forty-eight subjects were included in this study. Twenty-two subjects were classified as normal group and twenty-six subjects as patient group with tendinosis. MR examination was performed by 3 Tesla scanner using a 12 channel head coil. For relaxation times quantification, we used a sagittal 3D FLASH variable flip angle gradient echo UTE sequence (3D VFA-GE UTE) for T1 mapping and a sagittal Multi Echo Spin Echo sequence (MESE) for T2 mapping. Relaxation times were quantified using two different algorithms written in MATLAB. P value $<0.05$ was
\end{abstract} considered statistically significant.

\section{Results:}

Our results showed a statistically significant difference in T1 and T2 values for the normal group compared to the patient group ( $<<0.05$ ). Mean values of T1 and T2 were $571.69 \mathrm{~ms}$ and $24.16 \mathrm{~ms}$ for the normal group and $818.10 \mathrm{~ms}$ and $32.43 \mathrm{~ms}$ for the patient group, respectively. Results reported no correlation ( $\mathrm{r}=0.193)$ for $\mathrm{T} 1$ mapping and a positive significant moderate correlation $(\mathrm{r}=0.542, \mathrm{p}=0.000)$ for $\mathrm{T} 2$ mapping between the normal and patient groups. T1 and T2 showed no correlation in the normal group $(\mathrm{r}=0.091, \mathrm{p}=0.489)$ and a positive significant weak correlation in the patient group $(\mathrm{r}=0.263, \mathrm{p}=0.048)$.

\section{Conclusion:}

We concluded that $\mathrm{T} 1$ and $\mathrm{T} 2$ relaxation times are relatively sensitive to diagnosis degenerative changes in the $\mathrm{AT}$ and $\mathrm{T} 1$ is more sensitive to AT tendinosis compared to $\mathrm{T} 2$.

Keywords: Achilles tendon, Tendinosis, MRI, T1 and T2 mapping, Sagittal morphological image, Scanning parameters.

\begin{tabular}{ll|l|l} 
Article History & Received: April 30, 2019 & Revised: September 24, 2019 & Accepted: September 26, 2019
\end{tabular}

\section{INTRODUCTION}

Injuries of the Achilles Tendon (AT) are painful and can seriously hamper the activities of daily life. They may arise because of a variety of degenerative changes in the tendon composition including an increase in stiffness of the interfascicular matrix, an increase in collagen cross-linking and a reduction in collagen fibril crimp angle [1 - 3].

\footnotetext{
* Address correspondence to this author at El Manar University, Higher Institute of Medical Technologies of Tunis, Laboratory of Research in Biophysics and Medical Technologies, 09, Rue Doctor Zouheir Safi -1006 Tunis, Tunisia; Tel: 25513128; E-mail: zeinebtbini@mail.com
}

In the early stages of AT degeneration, it is difficult to detect the small morphological changes using ultrasound or conventional Magnetic Resonance Imaging (MRI). Relaxations Times such as $\mathrm{T} 1$ and $\mathrm{T} 2$ are sensitive to alteration or disruption of the Extracellular Matrix (ECM). Recently, quantitative assessment of AT injuries using biochemical sensitive MRI techniques presents the best opportunity to diagnose early microscopic degenerative changes of AT and follow-up of patients after treatment.

In the present work, we studied the biochemical changes in the components of AT using parametric MRI techniques. The goal was to measure and compare $\mathrm{T} 1$ and $\mathrm{T} 2$ relaxation times 
in normal people and patients with tendinosis and to evaluate their ability in the early diagnosis of AT tendinosis.

\section{MATERIALS AND METHODS}

\subsection{Study Group}

This prospective study was approved by the local Ethics Committee. Verbal and written informed consent was obtained from each patient.

A total of forty-eight subjects were scanned between January 2017 and January 2018 (27 right ankles and 21 left ankles). Twenty-two subjects (female, 13; male, 9; right, 12; left, 10; mean age, 37.9 \pm 16.6 years; Body Mass Index (BMI), $23.5 \pm 3.7 \mathrm{~kg} / \mathrm{m}^{2}$ ) were classified as the normal group upon clinical and ultrasound assessment. Twenty-six subjects (female, 20; right, 15; left, 11; male, 6; mean age, 49.31 \pm 14.66 years; BMI, $29.73 \pm 5.86 \mathrm{~kg} / \mathrm{m}^{2}$ ) were classified as the patient group with Achilles tendon tendinosis.

Inclusion criteria for the normal group were; no history of an ankle injury, no surgery, no infection or chronic disease. Exclusion criteria include a history of Achilles tears or major ankle trauma. For patients group, the inclusion criteria were chronic tendinopathy and mid-portion tendinosis of the Achilles tendon. The exclusion criteria were peritendinitis, rupture and traumatic Achilles pathology. The general exclusion criteria for normal and patient group were claustrophobe subjects and pregnant women.

The clinical and ultrasound assessment were performed by a musculoskeletal radiologist with 20 years of experience in musculoskeletal ultrasound. Ultrasound was performed in longitudinal and transverse orientation using color Doppler mode.

\subsection{MR Examination and Pulse Sequences}

MR examination was performed using a 3 Tesla scanner (Magnetom Verio, Siemens Erlangen, Germany) equipped with a 12 channel head coil. Subjects were positioned in the isocenter of the magnet, feet-first, supine position and both feet in flexion position but only one foot was placed in the coil. Patients were asked to stop all hard physical activities for 24 hours before the MR examination; also they stayed on rest a period of 20 minutes before MR imaging.

Table 1. Details of the scanning parameters for the morphological and parametric MRI sequences.

\begin{tabular}{|c|c|c|c|c|}
\hline $\begin{array}{l}\text { Sequences } \\
\text { Parameters }\end{array}$ & 2D TSE PD FAT-SAT ${ }^{a}$ & 2D TSE PD FAT-SAT ${ }^{b}$ & 3D VFA-GE UTE ${ }^{\mathrm{c}}$ & 2D MESE ${ }^{\mathrm{d}}$ \\
\hline Acquisition plan & Sagittal & axial & Sagittal & Sagittal \\
\hline Repetition time (ms) & 3000 & 3860 & 11 & 1500 \\
\hline Echo time (ms) & 27 & 34 & 1.45 & $8.1,16.2,24.3,32.4,40.5$ \\
\hline Flip angle $\left(^{\circ}\right)$ & 147 & 150 & 5,40 & 180 \\
\hline Field of view $(\mathrm{mm} * \mathrm{~mm})$ & $220 * 220$ & $273 * 330$ & $280 * 280$ & $280 * 280$ \\
\hline Bandwidth (Hz /pixel) & 246 & 250 & 425 & 250 \\
\hline Matrix & $384 * 326$ & $191 * 256$ & $410 * 512$ & $205 * 256$ \\
\hline Resolution & $384 * 384$ & $212 * 256$ & $512 * 512$ & $256 * 256$ \\
\hline Slice thickness (mm) & 3 & 3.5 & 1.19 & 3 \\
\hline Acquisition time (min: sec) & $3: 03$ & $2: 31$ & $3: 10$ & 2.5 \\
\hline
\end{tabular}

a sagittal Turbo Spin Echo Protons Density Fat-Sat sequence, ${ }^{\mathrm{b}}$ axial Turbo Spin Echo Protons Density Fat-Sat sequence, ${ }^{\mathrm{c}}$ sagittal 3D FLASH variable flip angle gradient echo UTE sequence, ${ }^{d}$ sagittal Multi Echo Spin Echo sequence.
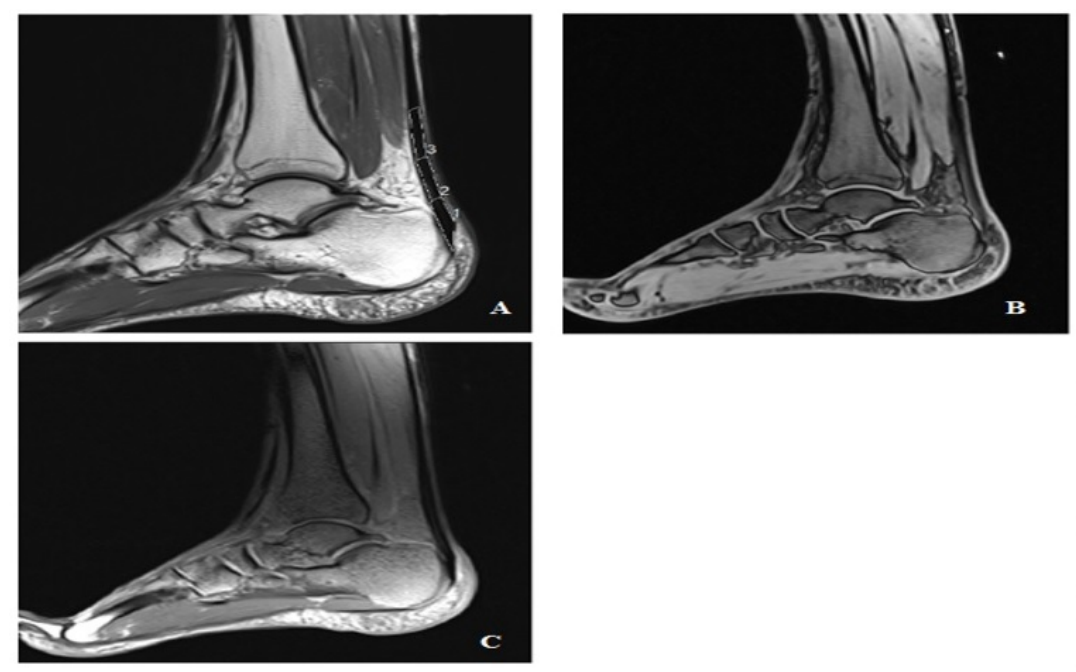

Fig (1). Sagittal morphological image, T1 and T2 images of the Achilles tendon of a 27-year-old volunteer. A. Sagittal morphological image, B. T1 image, C. T2 image. 
The morphological MR protocol of AT includes sagittal Turbo Spin Echo Protons Density Fat-Sat sequence (TSE PD FS) and axial TSE PD FS sequence. For quantification of relaxation times, we used a sagittal 3D FLASH variable flip angle gradient echo UTE sequence (3D VFA-GE UTE) for T1 mapping and a sagittal Multi Echo Spin Echo sequence (MESE) for T2 mapping. Table 1 shows the scanning parameters.

\subsection{Data Analysis}

Morphological evaluation of tendons was performed on sagittal and axial TSE PD FS images by a radiologist expert in musculoskeletal MRI (20 years of experience) to classify AT diseases and to confirm the inclusion and exclusion criteria for all the subjects. The evaluation was done using Siemens's workstation with syngo software.

$\mathrm{T} 1$ and $\mathrm{T} 2$ values were measured with respect to three Regions Of Interest (ROI) (Insertion Tendon (INS), MidPortion (MID), and Musculotendinous Junction (MJT)) drawn within each AT (Fig. 1). The length of each region is defined as a third of the total AT length, measured from the most proximal to the most distal [4].

$\mathrm{T} 1$ and $\mathrm{T} 2$ relaxation times values were quantified using two self-developed algorithms written in MATLAB (MATLAB 2014). The analysis algorithm is executed offline on sagittal images obtained with our quantitative T1 and T2 protocol. T1 and T2 maps were calculated on a voxel-by-voxel basis.

\subsubsection{T1 Calculation}

$\mathrm{T} 1$ values are estimated by a linear regression analysis from images acquired with 3D VFA-GE UTE according to the common Ernst equation for gradient-echo sequences:

$$
\mathrm{GE}_{\text {signal }}=\mathrm{M}_{0} \times \frac{\sin (\alpha) \times\left(1-\exp \left(-\frac{\mathrm{TR}}{\mathrm{T}_{1}}\right)\right)}{1-\cos (\alpha) \times \exp \left(-\frac{\mathrm{TR}}{\mathrm{T}_{1}}\right)}
$$

Where $\mathrm{T} 1$ is the longitudinal relaxation time, TR is the repetition Time, $\mathrm{M}$ is the initial magnetization at $\mathrm{t}=0$ and $\alpha$ is the flip angle.

We noted $\mathrm{Z}=\exp \left(-\frac{\mathrm{TR}}{\mathrm{T}_{1}}\right)$ and $\mathrm{A}=\mathrm{M} 0$ to simplify the notation. So, equation 1 becomes:

$$
\mathrm{GE}_{\text {signal }}=\frac{\mathrm{A} \times(1-\mathrm{Z}) \times \sin (\alpha)}{1-(\mathrm{Z} \times \cos (\alpha))}
$$

Equation 2 can be readjusted into a linear regression (eq. 3):

$$
\frac{\mathrm{GE}_{\text {signal }}}{\sin (\alpha)}=\mathrm{A} \times(1-\mathrm{Z})+\mathrm{Z} \times \frac{\mathrm{GE}_{\text {signal }}}{\tan (\alpha)}
$$

$\mathrm{A}$ and $\mathrm{Z}$ can be determined from the linear relationship between $\frac{\mathrm{GE}_{\text {signal }}}{\sin (\alpha)}$ and $\frac{\mathrm{GE}_{\text {signal }}}{\tan (\alpha)}$. We traced the curve based on the measurements as $\frac{\mathrm{GE}_{\text {signal }}}{\sin (\alpha)}=\mathrm{f}\left(\frac{\mathrm{GE}_{\text {signal }}}{\tan (\alpha)}\right) . \quad \mathrm{T} 1$ values were calculated from the slope of the curve using the following equation:

$$
\mathrm{T} 1=\frac{-\mathrm{TR}}{\ln (\text { slope })}
$$

\subsubsection{T2 Calculation}

T2 values are estimated by a monoexponential curve analysis from images acquired with MESE sequence according to the equation:

$$
\mathrm{SE}_{\text {signal }}=\mathrm{M}_{0} \exp \left(\frac{-T E}{T 2}\right)
$$

Where $\mathrm{M} 0$ is the initial magnetization at $\mathrm{t}=0$, TE is the echo time, and T2 is the transverse relaxation time constant. T2 values are calculated by least-squares fitting of equation 5 . We drew ROI's in the AT in one image, and propagated it to all images with variable TE. The mean intensities of pixels in each ROI's were used to plot T2 curve (equation. 6):

$$
\mathrm{S}=\mathrm{f}(\mathrm{TE})
$$

Where $\mathrm{S}$ refers to the SEsignal and TE is the echo time. T2 values were measured through the monoexponential fitting of the equation 7 :

$$
\left.Y=A \times \exp \left(\frac{-T E}{B}\right)\right)+C
$$

Where $\mathrm{A}$ is the signal intensity at $\mathrm{t}=0 \mathrm{~ms}, \mathrm{~B}$ refers to $\mathrm{T} 2$ and $\mathrm{C}$ is the offset.

\subsection{Statistical Analysis}

A paired sample T-test was realized to calculate the mean and SD of $\mathrm{T} 1$ and $\mathrm{T} 2$ relaxation times values and to compare the difference between the groups. Person correlation analysis was performed to compare $\mathrm{T} 1$ and $\mathrm{T} 2$ values of $\mathrm{AT}$ for the normal and patients group. The correlation coefficient $r$ indicates a very strong correlation $(\mathrm{r}=0.80$ to 1.00$)$, a strong correlation ( $\mathrm{r}=0.60$ to 0.79 ), a moderate correlation ( $\mathrm{r}=0.40$ to $0.59)$, a weak correlation ( $r=0.20$ to 0.39 ) or no correlation $(\mathrm{r}<0.20)$. We drew T1 and T2 curves and performed linear regression on each of these plots. The slope of each regression line was used as an indicator of the dynamic range for each parameter. Then, we calculated the coefficient of determination $\left(R^{2}\right)$ where $R^{2}$ equal to 1 indicates a very high correlation. Statistical evaluation was performed with SPSS 15.0 (SPSS, Chicago, Illinois, USA). P-value of less than 0.05 was considered to be statistically significant.

\section{RESULTS}

The results of T1 and T2 imaging of the Achilles tendon of all 48 subjects were analyzed according to the normal and patient group. Axial and sagittal morphological images, T1 and T2 images of the AT are shown in Fig. (1).

T1 and T2 mean values in each ROI of the AT for normal and patients group are summarized in Table 2. Our results show a statistically significant difference in $T 1$ and $T 2$ values in the normal group compared to the patient group $(\mathrm{p}<0.05)$. Means values of T1 and T2 in the whole AT were $571.69 \mathrm{~ms}$ and $24.16 \mathrm{~ms}$ for the normal group and $818.10 \mathrm{~ms}$ and 32.43 $\mathrm{ms}$ for the patient group. There were no significant differences in $\mathrm{T} 1$ and $\mathrm{T} 2$ values between the different regions of interest 
$(p>0.05)$ for the normal group. Whereas, there was a significant difference in the patient group.

The analysis of the variation in $\mathrm{T} 1$ and $\mathrm{T} 2$ values between the normal and patient group showed no correlation for T1 $(\mathrm{r}=0.193)$. Whereas, $\mathrm{T} 2$ revealed a positive significant moderate correlation $(\mathrm{r}=0.542, \mathrm{p}=0.000)$.

For the normal group, there was no correlation between $\mathrm{T} 1$ and $\mathrm{T} 2(\mathrm{r}=0.091, \mathrm{p}=0.489)$. The plot of $\mathrm{T} 1$ and $\mathrm{T} 2$ shows a slope equal to 0.0175 with a coefficient of determination $\mathrm{R}^{2}$ equal to 0.22 . (Fig. 2).

For the patient group, Person's correlation analysis showed a positive weak correlation between $\mathrm{T} 1$ and $\mathrm{T} 2(\mathrm{r}=0.263$, $\mathrm{p}=0.048)$. The plot of $\mathrm{T} 1$ and $\mathrm{T} 2$ shows a slope equal to -0.002 $\left(\mathrm{R}^{2}=0.0087\right)$ (Fig. 3).

Table 2. Mean T1 and T2 values in IN, MID and MTJ regions of interest for volunteers and patient group.

\begin{tabular}{|c|c|c|c|c|}
\hline \multirow{2}{*}{-} & \multicolumn{2}{|c|}{ T1 value (ms) } & \multicolumn{2}{c|}{ T2 value (ms) } \\
\cline { 2 - 5 } & Volunteers & Patients & Volunteers & Patients \\
\hline $\mathrm{IN}^{\mathrm{a}}$ & 573,51 & $798.55 \pm 404.03$ & 24,86 & $35.33 \pm 11.11$ \\
\hline $\mathrm{MID}^{\mathrm{b}}$ & 571,63 & $792.53 \pm 401.43$ & 23,25 & $35.33 \pm 11.11$ \\
\hline $\mathrm{MTJ}^{\mathrm{c}}$ & 569,92 & $787.29 \pm 397.43$ & 24,36 & $35.05 \pm 12.35$ \\
\hline
\end{tabular}

a: Insertion Tendon (INS), b: mid-portion (MID), c: Musculotendinous Junction (MJT), ms: millisecond.

$\mathrm{SD}=$ standard deviation; $\mathrm{TST}=$ tuberculin skin test

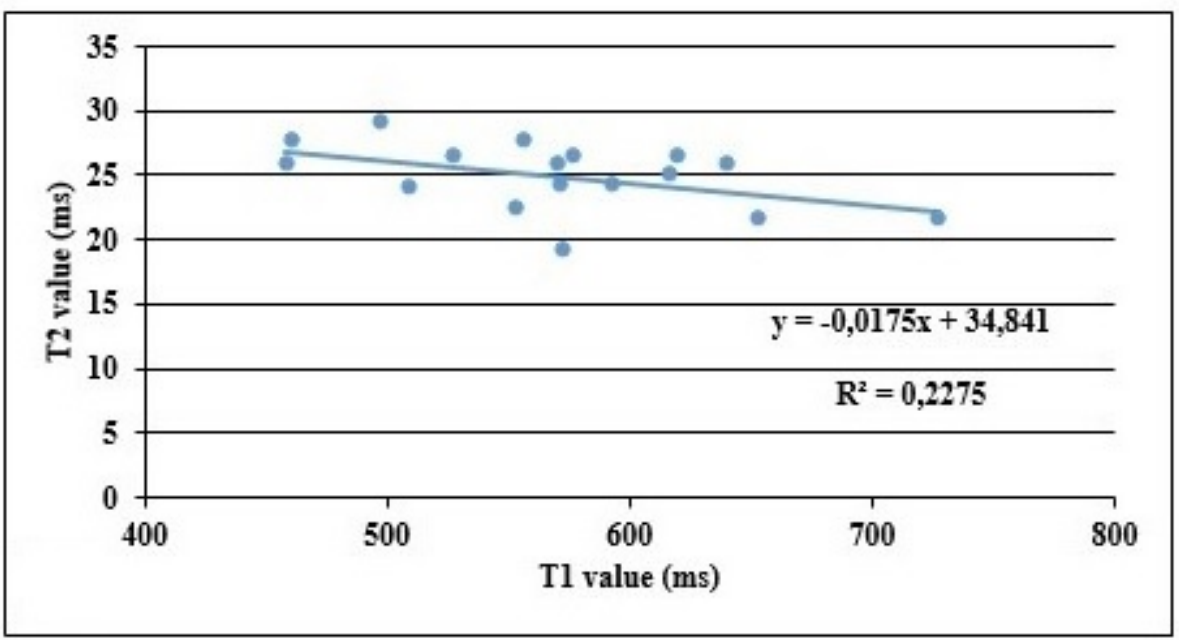

Fig (2). Plot curves of $\mathrm{T} 1$ and $\mathrm{T} 2$ for normal group.

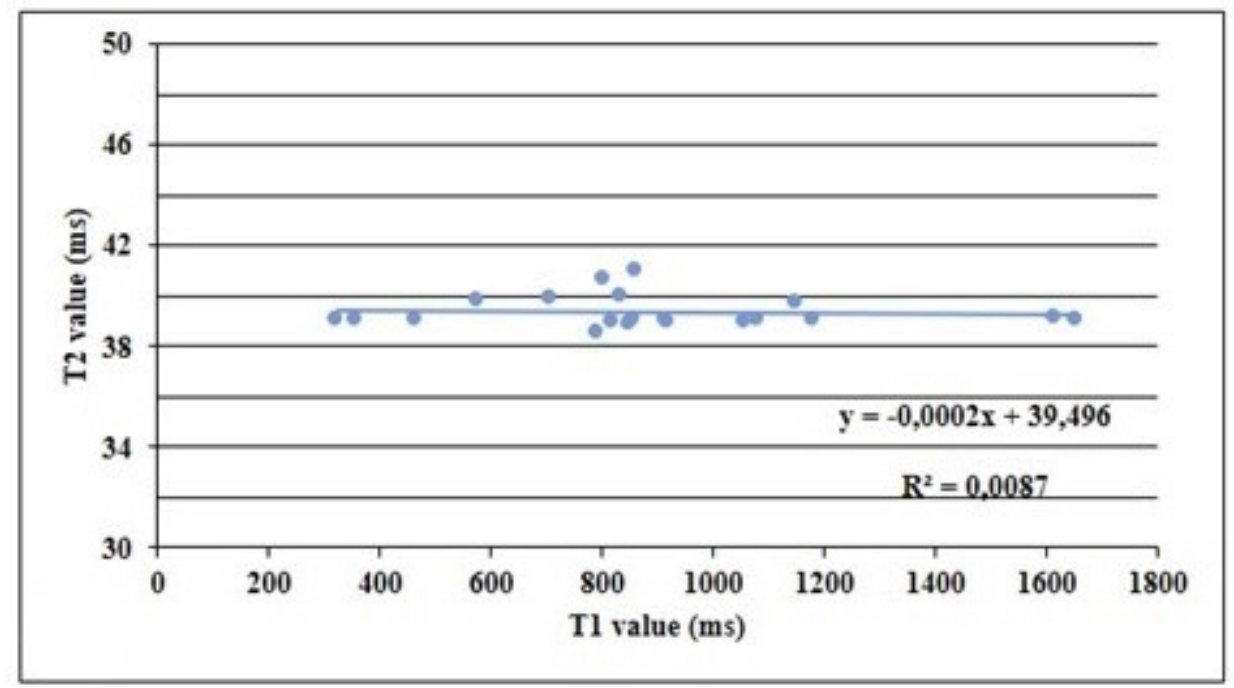

Fig (3). Plot curves of $\mathrm{T} 1$ and $\mathrm{T} 2$ for patient group.

\section{DISCUSSION}

$\mathrm{T} 1$ and T2 mapping techniques have been used to analyze significant changes in the structural integrity of tendon [5 - 15].
In this work, Achilles tendon of the same subjects was imaged using the two quantitative MRI techniques (T1 and T2 mappings), and results of the patient group were compared 
with those of the normal group to define the ability of these techniques for the diagnosis of AT tendinosis.

Our study results indicate significant differences in $\mathrm{T} 1$ and $\mathrm{T} 2$ mean values between the normal and patient group. These differences may reflect degeneration within the matrix of the tendon. The variation in $\mathrm{T} 1$ and $\mathrm{T} 2$ values between the normal and patient group, indicated no correlation for $\mathrm{T} 1$ and a positive significant moderate correlation for $\mathrm{T} 2$. The correlation analysis of $\mathrm{T} 1$ and $\mathrm{T} 2$ revealed no correlation for the normal group and a positive significant weak correlation for the patient group.

Ulrich Grosse et al. have found $\mathrm{T} 1$ of $658.4 \mathrm{~ms} \pm 21.8 \%$ for asymptomatic non-tendinopathic tendon and $856.1 \mathrm{~ms} \pm$ $28.4 \%$ for symptomatic tendons using 3D-FLASH Ultrashort Echo time (UTE) sequence with variation of the flip angle at 3 Tesla [16]. Juras and Apprich investigating the feasibility of T2 mapping, have reported that the mean T2 in INS, MID and MTJ were $21.50 \mathrm{~ms}, 13.13 \mathrm{~ms}$ and $14.36 \mathrm{~ms}$ respectively [6].

For T1 mapping, our results showed an increase in T1 values as the degenerative change increased in the Achilles tendon of the patient group. Wright et al., studied patient with spondylarthritis at the Achilles tendon, and indicated that T1 mapping has the potential to identify tendon abnormality earlier in comparison to conventional MR sequences by maximizing the sensitivity to subtle changes in the tendon structure [17].

In this study, T2 relaxation times values showed a moderate correlation between the normal and patient group. Edmund Ganal et al., evaluated the efficacy of T2 mapping in detecting differences in the Supraspinatus Tendon (SST) and concluded that T2 mapping is an accurate non-invasive method to identify quantitatively early rotator cuff pathology and the tear group exhibited significantly higher mean $\mathrm{T} 2$ values than the tendinosis and asymptomatic groups [10].

Analysis of T1 and T2 revealed no correlation for the normal group and a positive significant weak correlation for the patient group. This difference in results can be explained by the differences in scanning parameters and tissue type.

$\mathrm{T} 1$ and $\mathrm{T} 2$ values in AT suggested a significant variation between the three anatomical Regions Of Interest (ROI). The regional variation in $\mathrm{T} 1$ and $\mathrm{T} 2$ values is very clear for the patient group compared to the normal group. The difference in our results and previous published studies can be explained by the differences in scanning parameters, MRI exam conditions and population cohort. Moreover, the results can be influenced by several factors including the magnetic field strength of the MR system, the fitting algorithms and the un-voided experimental noises.

The movement of protons in Achilles tendon is reliant on the orientation of the collagen fibers. When the collagen fibers are oriented $55^{\circ}$ to the $\mathrm{B} 0$, dipolar interactions reduce and $\mathrm{T} 2$ is increased, the so-named the magic angle effect $[18,19]$. Henkel man et al. report that the mean T2 of in vitro dog Achilles tendon increases from 7 to $23 \mathrm{~ms}$ when the orientation is changed from $0^{\circ}$ to $55^{\circ}$ [19]. Generally, the magic angle effect is interpreted as an artifact and results in an increased signal intensity in Achilles tendon on sequences with low TE such as T1-, PD- and GRE sequences [20]. In our study, the magic angle effect is minimized since we compared the same region in all the subjects. The UTE sequences are specifically sensitive to the magic angle effect because of the short TE [21]. Therefore, it is essential that the Achilles tendon is placed parallel to the magnetic field when using UTE sequences. This problem is minimized in our study since we positioned all the subjects in supine position and both feet in flexion position parallel to the axis of the table.

Our study is exposed to some limitations. First, the number of subjects is small. Second, the population is heterogeneous and not matched for age, BMC and gender which limits the accuracy of the analysis of the results. Third, this study needs more histological information. Further studies with a larger patient group with histologic correlation are helpful for the evaluation of the reliability of quantitative MR techniques in the early diagnosis of mechanical Achilles pathologies.

\section{CONCLUSION}

We concluded that $\mathrm{T} 1$ and $\mathrm{T} 2$ relaxation times are relatively sensitive to degenerative changes in the AT and $\mathrm{T} 1$ and $\mathrm{T} 2$ are positively correlated just in the patient group. $\mathrm{T} 1$ relaxation time is more sensitive to AT tendinosis compared to $\mathrm{T} 2$.

\section{AUTHORS' CONTRIBUTION}

Tbini Zeineb contributed to the conception of the work, and helped in conducting the study, analyzing the patient data, interpreting the results, and revising the draft. Tbini zeineb also approved the final version of the manuscript, and assisted in drafting and revising the draft, and showed agreement to the conent of the work and was a major contributor in writing of the manuscript.

Mars Mokhtar contributed to the conception of the work, and helped in conducting the study, adjusting MRI exam parameters, interpreting the patient data, revising the draft, approving the final version of the manuscript, and gave agreement to the content of the work.

Mouna Chelli Bouaziz performed the classification of patients and controlled the MRI exam, and also helped in revising the draft, approving the final version of the manuscript. He also showed agreement to the content of the work.

\section{ETHICS APPROVAL AND CONSENT TO PARTICIPATE}

The study was approved by Ethics Committee of Kassab Orthopedic Institute, Tunis El Minar University, Tunisia with approval number : CE-IMKO 2015/105.

\section{HUMAN AND ANIMAL RIGHTS}

No animals were used in this research. All human research procedures followed were in accordance with the ethical standards of the committee responsible for human experimentation (institutional and national), and with the Helsinki Declaration of 1975, as revised in 2013. 


\section{CONSENT FOR PUBLICATION}

Verbal and written informed consent was obtained from each patient.

\section{AVAILABILITY OF DATA AND MATERIALS}

Not applicable

\section{FUNDING}

None.

\section{CONFLICT OF INTEREST}

The authors declare no conflict of interest, financial or otherwise.

\section{ACKNOWLEDGEMENTS}

Declared none.

\section{REFERENCES}

[1] Laura Chernak Slane \& Jack Martin \& Ryan De Wall \& Darryl Thelen \& Kenneth Lee. Quantitative ultrasound mapping of regional variations in shear wave speeds of the aging Achilles tendon 2017; 27(2): 474-82

[2] Thorpe CT, Udeze CP, Birch HL, Clegg PD, Screen HR. Capacity for sliding between tendon fascicles decreases with ageing in injury prone equine tendons: A possible mechanism for age-related tendinopathy? Eur Cell Mater 2013; 25: 48-60.

[http://dx.doi.org/10.22203/eCM.v025a04] [PMID: 23300032]

[3] Couppé C, Hansen P, Kongsgaard M, et al. Mechanical properties and collagen cross-linking of the patellar tendon in old and young men. J Appl Physiol 2009; 107(3): 880-6.

[http://dx.doi.org/10.1152/japplphysiol.00291.2009]

[PMID: 19556458]

[4] Juras Apprich. Bi-exponential T2 analysis of healthy and diseased Achilles tendons: an in vivo preliminary magnetic resonance study and correlation with clinical score. Eur Radio 201323(10): 2814-22. [http://dx.doi.org/10.1007/s00330-013-2897-8]

[5] Fukawa T, Yamaguchi S, Watanabe A, et al. Quantitative assessment of tendon healing by using MR T2 mapping in a rabbit achilles tendon transection model treated with platelet-rich plasma. Radiology 2015; 276(3): 748-55

[http://dx.doi.org/10.1148/radiol.2015141544] [PMID: 25816105]

[6] Juras V, Apprich S, Pressl C, et al. Histological correlation of $7 \mathrm{~T}$ multi-parametric MRI performed in ex-vivo Achilles tendon. Eur J Radiol 2013; 82(5): 740-4.

[http://dx.doi.org/10.1016/j.ejrad.2011.09.022] [PMID: 22177325]

[7] Marshall Clare Howarth, Larkman David J, Herlihy Amy H, Oatridge Angela, Bydder Graeme M. Contrast-enhanced magic-angle MR imaging of the achilles tendon. AJR Am J Roentgenol 2002; 179(1):
187-92.

[8] Oatridge A, Herlihy A, Thomas R W, et al. Magic Angle Imaging of the Achilles Tendon in Patients with Chronic Tendonopathy. Clin Radiol 2003; 58(5): 384-8.

[9] Adam W. Anz, Erin P. Lucas, Eric K. Fitzcharles, Rachel K. Surowiec, Peter J. Millett, Charles P. Ho, MRI T2 mapping of the asymptomatic supraspinatus tendon by age and imaging plane using clinically relevant subregions. Eur J Radiol 2014; 83(5): 801-5. [http://dx.doi.org/10.1016/j.ejrad.2014.02.002]

[10] Ganal E. Ho CP, Wilson KJ et al. Quantitative MRI characterization of arthroscopically verified supraspinatus pathology: Comparison of tendon tears, tendinosis and asymptomatic supraspinatus tendons with T2 mappingKnee Surg Sports Traumatol Arthrosc 2016; 24(7): 2216-4.

[11] Tallon C. Nicola maffulli, and stanley W. B. Ewen, ruptured achilles tendons are significantly more degenerated than tendinopathic tendons. Med Sci Sports Exerc. 2001; 33: pp. (12)1983-90.

[12] Zheng S, Xia Y. Multi-components of T2 relaxation in ex vivo cartilage and tendon. J Magn Reson 2009; 198(2): 188-96. [http://dx.doi.org/10.1016/j.jmr.2009.02.005] [PMID: 19269868]

[13] Kijowski Richard, Wilson John J, Liu Fang. Bicomponent ultrashort echo time T2* analysis for assessment of patients with patellar tendinopathy. J Magn Reson Imaging 2017; 46(5): 1441-7.

[14] Stefan K, Lothar R. Single-slice mapping of ultrashort T(2). J Magn Reson 210(1): 133-6.

[http://dx.doi.org/10.1016/j.jmr.2011.02.003] [PMID: 21353799]

[15] Wilson KJ, Surowiec RK, Johnson NS, Lockard CA, Clanton TO, Ho CP. T2* mapping of peroneal tendons using clinically relevant subregions in an asymptomatic population. Foot Ankle Int 2017; 38(6): 677-83.

[http://dx.doi.org/10.1177/1071100717693208] [PMID: 28552042]

[16] Grosse U, Syha R, Hein T, Gatidis S et al. Diagnostic value of T1 and T2* relaxation times and off-resonance saturation effects in the evaluation of achilles tendinopathy by MRI at 3TJournal of Magnetic Resonance Imaging 2015; 41(4): 964-73.

[17] Wright Peter, Jellus Vladimir, McGonagle Dennis, Robson Matthew, Ridgeway John, Hodgson Richard. Comparison of two ultrashort echo time sequences for the quantification of T1 within phantom and human achilles tendon at 3 T. Magnetic Resonance in Medicine 2012; 68(4): 1279-84.

[18] Peto S, Gillis P, Henri VP. Structure and dynamics of water in tendon from NMR relaxation measurements. Biophys J 1990; 57(1): 71-84. [http://dx.doi.org/10.1016/S0006-3495(90)82508-X] [PMID: 2297563]

[19] Henkelman RM, Stanisz GJ, Kim JK, Bronskill MJ. Anisotropy of NMR properties of tissues. Magn Reson Med 1994; 32(5): 592-601. [http://dx.doi.org/10.1002/mrm.1910320508] [PMID: 7808260]

[20] Fullerton GD, Rahal A. Collagen structure: The molecular source of the tendon magic angle effect. J Magn Reson Imaging 2007; 25(2): 345-61. [Review].

[http://dx.doi.org/10.1002/jmri.20808] [PMID: 17260393]

[21] Bydder M, Rahal A, Fullerton GD, Bydder GM. The magic angle effect: A source of artifact, determinant of image contrast, and technique for imaging. J Magn Reson Imaging 2007; 25(2): 290-300. [http://dx.doi.org/10.1002/jmri.20850] [PMID: 17260400]

This is an open access article distributed under the terms of the Creative Commons Attribution 4.0 International Public License (CC-BY 4.0), a copy of which is available at: (https://creativecommons.org/licenses/by/4.0/legalcode). This license permits unrestricted use, distribution, and reproduction in any medium, provided the original author and source are credited. 